August 1992

MPI-Ph/92-61

TUM-TH-147/92

\title{
Modular Groups for Twisted Narain Models*
}

\author{
Jens Erler \\ Max-Planck-Institut für Physik \\ - Werner-Heisenberg-Institut — \\ Föhringer Ring 6 \\ Postfach 401212 \\ W - 8000 Munich 40 (Germany) \\ and \\ Michał Spaliński \\ Instytut Fizyki Teoretycznej \\ Uniwersytetu Warszawskiego \\ Warszawa (Polska)
}

\begin{abstract}
We demonstrate how to find modular discrete symmetry groups for $Z_{N}$ orbifolds. The $Z_{7}$ orbifold is treated in detail as a non-trivial example of a $(2,2)$ orbifold model. We give the generators of the modular group for this case which, surprisingly, does not contain $S L(2 ; \mathbf{Z})^{3}$ as had been speculated. The treatment models with discrete Wilson lines is also discussed. We consider examples which demonstrate that discrete Wilson lines affect the modular group in a non-trivial manner. In particular, we show that it is possible for a Wilson line to break $S L(2, \mathbf{Z})$.
\end{abstract}

*Supported by Deutsche Forschungsgemeinschaft 


\section{Introduction}

One of the main tasks of the string theorist today is to find the true vacuum configuration of the heterotic string. On one hand one should try to phenomenologically constrain possible vacua and on the other hand it is clearly important to understand the nature of the vacuum degeneracy and the dynamical effects which could lead to the determination of the ground state.

The space of string vacua is locally parametrized by moduli, which are marginal deformations of the underlying conformal field theory [1]. An intriguing feature of string compactifications is that the natural parametrizations of the moduli space label the points in a redundant way, since all physical quantities are invariant under the action of some discrete group acting on the moduli.

In the low energy effective theory the moduli correspond to vacuum expectation values of massless scalar fields which have flat potentials to all orders in perturbation theory [2, 3]. It would thus be of considerable phenomenological importance, if the degeneracy of the vacua described by these moduli could be lifted. One attempt [4, 5, 6] in this direction is based on the assumption that the modular discrete symmetry group derived in the framework of string perturbation theory remains unbroken after taking into account non-perturbative effects.

It is also interesting, that the modular group typically includes duality symmetries [7, 8, 9, 10], which relate small and large radii. Like all modular symmetries, it must be used to restrict the moduli space to a fundamental domain. Duality is a stringy property, not found in a point particle context. If signs of such a symmetry would survive down to low energies, they would constitute an indication of a compactified string theory at some level.

A simple yet quite rich class of compactifications which allow a detailed study of the moduli space are $Z_{N}$ orbifolds [11, 12], also referred to as twisted Narain compactifications [13, 14]. These models are parametrized by constant background fields which are interpreted as the orbifold moduli. Having the moduli explicitly in hand makes it possible to answer detailed questions about the structure of moduli space and in particular the discrete symmetries 15, 16, 17, 18. This is especially exciting since this class of models has very promising prospects for phenomenology.

Until recently the only examples where the modular group had been identified were tori and symmetric orbifolds in one and two dimensions [1, 7, 8, 9, 19, 20]. In this paper we investigate two compactifications, each serving as a representative of a large class of models. First we consider the $Z_{7}$ orbifold falling into the class of vacua with $(2,2)$ world sheet supersymmetry. Motivated by the results in [19, 20], where the modular group $S L(2 ; \mathbf{Z})$ was found, it was sometimes conjectured that this group is independently realized 
for any complex modulus which appears, so that the modular group would always be $S L(2 ; \mathbf{Z})$ to some power. This conjecture became known as the "maximal discrete symmetry hypothesis". This belief suffered a setback with the discovery that the modular group for the complex structure modulus of the mirror quintic was different [21]. Nevertheless, it was still speculated that the conjecture could be true in orbifold compactifications. For the $Z_{7}$ orbifold the symmetry group expected on the basis of the maximal discrete symmetry hypothesis would be $S L(2, \mathbf{Z})^{3}$. This is in fact not the case - instead we find a surprisingly rich structure, where $S L(2, \mathbf{Z})$ subgroups are not realized independently on each of the individual complex moduli associated with the three complex planes distinguished by the twist.

A related longstanding problem concerned the case where quantized Wilson lines are present. These models are of particular phenomenological interest, as three generation models of $S U(3) \times S U(2) \times U(1)^{n}$ can be constructed in this class [22]. The question whether a duality symmetry in moduli space can be defined has been questioned seriously, since it was realized [17] that the duality transformation rules from the torus applied to these cases lead to asymmetric orbifolds. Here we will show, for the $Z_{3}$ orbifold, that turning on discrete Wilson lines in fact breaks $S L(2 ; \mathbf{Z})$ to a subgroup.

Section 2 reviews the Narain model and its modular symmetries. Section 3 discusses symmetric $Z_{N}$ orbifolds of the Narain model, stressing various points which are important for further developments. It also discusses the modular symmetries of these models. Our main results are presented in sections 14 and 5, where the modular groups of the $Z_{7}$ orbifold and the $Z_{3}$ orbifold with a Wilson line background are described. Section 1 also contains a direct proof of the fact that $S L(2 ; \mathbf{Z})$ is the modular group for the $Z_{3}$ orbifold in two compact dimensions.

\section{The Narain model}

The Hilbert space of a Narain model [13, 14] is built by applying the standard mode creation operators to states of given momentum and winding. The states may therefore be labelled by the left and right momenta, associated with the holomorphic and antiholomorphic sectors of the world sheet CFT and by the mode numbers. The entire dependence on the moduli lies in the momenta labelling the ground state of a tower of integer-spaced states.

Narain models are obtained by compactifying $d$ coordinates on a torus, whose geometry is specified by an even, self-dual lattice with a Lorentzian metric. The set of such lattices is labelled by $d(d+16)$ continuous parameters, which may be interpreted in terms of expectation values of background fields: the lattice metric $g$, an antisymmetric tensor field $b$ and Wilson lines $a$.

In our case the lattices in $(16+d, d)$ dimensions are of relevance and can 
be described in terms of the spectra of the left and right momenta, which due to the compactification become discrete. The parametrization in terms of constant background fields reads円

$$
\begin{aligned}
& P_{L}=\left(m+(g-h) n-a^{T} C l, \quad l+a n\right)=\left(\begin{array}{cc}
p_{L} & , \tilde{p}_{L}
\end{array}\right), \\
& P_{R}=\left(m-(g+h) n-a^{T} C l, \quad 0 \quad\right)=\left(\begin{array}{cc}
p_{R}, & 0
\end{array}\right) \text {. }
\end{aligned}
$$

These expressions are understood in the lattice basis, so that the windings $n$, momenta $m$ and gauge quantum numbers $l$ are all integer valued vectors. In this formulae $g$ denotes the lattice metric (a $d \times d$ matrix) and $a$ is the Wilson line (a $16 \times d$ matrix). Furthermore we defined

$$
h=b+\frac{1}{2} a^{T} C a,
$$

where $b$ is the antisymmetric "axionic" background and $C$ denotes the $E_{8} \times E_{8}$ Cartan metric.

Dropping moduli independent oscillator contributions, the conformal dimensions are given by

$$
\begin{aligned}
& L_{0}=\frac{1}{4} p_{L} g^{-1} p_{L}+\frac{1}{2} \tilde{p}_{L} C \tilde{p}_{L}, \\
& \bar{L}_{0}=\frac{1}{4} p_{R} g^{-1} p_{R} .
\end{aligned}
$$

For many purposes it is convenient to consider the linear combinations,

$$
\begin{gathered}
H=L_{0}+\bar{L}_{0}, \\
P=L_{0}-\bar{L}_{0},
\end{gathered}
$$

which are interpreted as the world sheet energy and momentum, respectively. Since both quantities are quadratic in the quantum numbers, they can be written in the form

$$
\begin{aligned}
H & =\frac{1}{2} u^{T} \chi u, \\
P & =\frac{1}{2} u^{T} \eta u,
\end{aligned}
$$

where

$$
\begin{gathered}
u=\left(\begin{array}{c}
n \\
m \\
l
\end{array}\right), \\
\eta=\left(\begin{array}{ccc}
0 & \mathbf{1}_{d} & 0 \\
\mathbf{1}_{d} & 0 & 0 \\
0 & 0 & C
\end{array}\right),
\end{gathered}
$$

\footnotetext{
${ }^{1}$ In this and in the following two sections we set the Regge slope $\alpha^{\prime}$ to 1.
} 
and

$$
\chi=\left(\begin{array}{ccc}
\left(g+h^{T}\right) g^{-1}(g+h) & -h^{T} g^{-1} & \left(g+h^{T}\right) g^{-1} a^{T} C \\
-g^{-1} h & g^{-1} & -g^{-1} a^{T} C \\
C a g^{-1}(h+g) & -C a g^{-1} & C+C a g^{-1} a^{T} C
\end{array}\right)
$$

Thus $u$ is a $2 d+16$ component integer vector, while $\chi$ and $\eta$ are $2 d+16$ dimensional square matrices and $\mathbf{1}_{d}$ denotes the identity matrix in $d$ dimensions. The above formulation is useful, since all the moduli dependence resides in $\chi$, while $\eta$ is constant over moduli space.

Discrete symmetries in moduli space can be studied by looking for linear transformations of the quantum numbers, which preserve the spectrum. Such transformations can be written as

$$
u \longrightarrow S_{\Omega}(u)=\Omega^{-1} u .
$$

The requirement that $P$ should be preserved by this transformation translates to the following condition on the integer matrix $\Omega$ :

$$
\Omega^{T} \eta \Omega=\eta
$$

The group formed by such matrices will be denoted by $\mathcal{G}_{\mathcal{N}}$.

To be a symmetry of the spectrum, the transformation (2.11) must also leave $H$ invariant. Since $H$ depends on the moduli, invariance can only be achieved if the background is appropriately transformed. The required transformation law is

$$
\chi \longrightarrow S_{\Omega}(\chi)=\Omega^{T} \chi \Omega .
$$

This defines the action of $\mathcal{G}_{\mathcal{N}}$ on the moduli.

\section{Twisted Narain models}

The untwisted sector of the orbifold Hilbert space is obtained by a projection of the Hilbert space of a Narain compactified heterotic string and adding twisted sectors needed to maintain modular invariance. The orbifold projection is defined in terms of an automorphism group of the Narain lattice 112, 16, 23. To construct a $Z_{N}$ orbifold [1] one may proceed by choosing an element $\mathcal{R}$ of $\mathcal{G}_{\mathcal{N}}$ (the defining matrix) which generates a $Z_{N}$ group and projecting onto invariant states.

The action of the twist on the quantum numbers is given by

$$
u \longrightarrow u^{\prime}=\mathcal{R} u \text {. }
$$

For the Narain lattice to admit such an automorphism group, the background has to satisfy the consistency condition (see (2.6))

$$
\mathcal{R}^{T} \chi \mathcal{R}=\chi
$$


This is a set of homogenous linear equations determining the specific form of the background which admits a given twisting. The solutions to this equation are parametrized by the moduli.

Requiring that the orbifold be left - right symmetric restricts the possible form of the defining matrix $\mathcal{R}$. One must have

$$
n \longrightarrow n^{\prime}=Q n
$$

where $Q$ is the twist matrix in the lattice basis [12]. One must also require that the twist acts trivially on $\tilde{p}_{L}$, since the corresponding (vanishing) components of $P_{R}$ are not affected by the twist. It then follows that

$$
l \longrightarrow l^{\prime}=l+a\left(\mathbf{1}_{d}-Q\right) n .
$$

This way one arrives at the conclusion that $\mathcal{R}$ must have the form

$$
\mathcal{R}=\left(\begin{array}{lll}
Q & 0 & 0 \\
\alpha & \beta & \gamma \\
a\left(\mathbf{1}_{d}-Q\right) & 0 & \mathbf{1}_{d}
\end{array}\right)
$$

where $\alpha, \beta, \gamma$ are matrices whose form will be determined by the requirement that $\mathcal{R}$ should belong to $\mathcal{G}_{\mathcal{N}}$. The use of $(2.12)$ leads to the result that

$$
\begin{aligned}
\alpha & =Q^{*} \delta+\frac{1}{2} a^{T} C a\left(\mathbf{1}_{d}-Q\right)+\frac{1}{2}\left(\mathbf{1}_{d}-Q^{*}\right) a^{T} C a \\
\beta & =Q^{*} \\
\gamma & =\left(\mathbf{1}_{d}-Q^{*}\right) a^{T} C
\end{aligned}
$$

where $\delta$ is an antisymmetric integer matrix, which is not determined by the condition (2.12). The $*$ denotes the inverse transpose of a matrix, i.e. $\left.A^{*} \equiv\left(A^{T}\right)^{(}-1\right)$.

Since $\mathcal{R}$ has to be an integer matrix in order to have a well defined meaning on the quantum numbers, it follows from the above that the Wilson line has to satisfy

$$
\begin{gathered}
Q^{*} \delta+\frac{1}{2} a^{T} C a\left(\mathbf{1}_{d}-Q\right)+\frac{1}{2}\left(\mathbf{1}_{d}-Q^{*}\right) a^{T} C a \in \mathbf{Z}, \\
a\left(\mathbf{1}_{d}-Q\right) \in \mathbf{Z} .
\end{gathered}
$$

These conditions were originally found by Ibañez et al. [12].

One must also require that the defining matrix should preserve $\chi$ in the sense of (3.2). This yields

$$
\begin{gathered}
Q^{T} g Q=g, \\
Q^{T} b Q=b+\delta,
\end{gathered}
$$


where $\delta$ is the antisymmetric integer matrix appearing in (3.6). These equations determine the backgrounds in terms of independent deformation parameters (the moduli). Of course (3.10) may not admit a solution with a non-vanishing $\delta$. This is in fact the case in all the examples discussed in this paper. Note finally that (3.2) imposes no conditions on the Wilson lines. There can however be additional conditions on the Wilson lines coming from modular invariance [11] (level matching).

Even though twisted Narain models are constructed directly from the untwisted ones, not all the modular discrete symmetries of the orbifold moduli space are inherited by the orbifold. Only those symmetries survive, which are compatible with the orbifold projection, as will now be reviewed.

The conditions for modular discrete symmetries in orbifold models were studied in [15, 16, 17]. The basic requirement to be imposed is that symmetry transformations map physical states at a reference background to physical states at the transformed background. By physical states we mean here twist invariant states of the model under consideration[2]. This is the case for those $\Omega \in \mathcal{G}_{\mathcal{N}}$, which preserve the $Z_{N}$ group in the sense that

$$
\Omega \mathcal{R} \Omega^{-1}=\mathcal{R}^{p}
$$

for some $p=1, \ldots, N-1$. This condition selects a subgroup of $\mathcal{G}_{\mathcal{N}}$, which is the discrete symmetry group of the orbifold compactification. This group will be referred to as $\mathcal{G}_{\mathcal{O}}$, defined as

$$
\mathcal{G}_{\mathcal{O}}:=\left\{\Omega \in \mathcal{G}_{\mathcal{N}} \mid \Omega \mathcal{R} \Omega^{-1}=\mathcal{R}^{p}, p \in \mathbf{Z}\right\} .
$$

The condition (3.11) may be called the compatibility condition for the discrete transformation specified by $\Omega$. It guarantees that transformations induced by elements of $\mathcal{G}_{\mathcal{O}}$ are modular symmetries of the orbifold compactification.

Not all the elements belonging to $\mathcal{G}_{\mathcal{O}}$ induce non-trivial transformations in moduli space. For example the defining matrix $\mathcal{R}$ itself trivially satisfies (3.11) with $p=1$ and by construction leaves the background unchanged. Thus, the representation of $\mathcal{G}_{\mathcal{O}}$ on the orbifold moduli space is not faithful. It is therefore convenient to define an equivalence relation in $\mathcal{G}_{\mathcal{O}}$ by declaring all elements, which induce the same transformation of the moduli, to be equivalent. The group of discrete symmetries acting on the moduli space may then be defined as the quotient of $\mathcal{G}_{\mathcal{O}}$ by the above equivalence. To avoid misunderstandings, this quotient group will be denoted by $\tilde{\mathcal{G}}_{\mathcal{O}}$.

\footnotetext{
${ }^{2}$ Note that in [17] a more general situation is considered, where the physical states of one model are mapped to physical states of another yet equivalent model.
} 
The canonical duality transformation for the Narain model is the transformation induced by

$$
\Omega=\left(\begin{array}{ccc}
0 & \mathbf{1}_{d} & 0 \\
\mathbf{1}_{d} & 0 & 0 \\
0 & 0 & \mathbf{1}_{16}
\end{array}\right)
$$

In the orbifold case the above transformation is typically not modular. Instead, one has to consider the following family of transformations [15, 16],

$$
\Omega_{S}:=\left(\begin{array}{ccc}
0 & W^{-1} & 0 \\
W^{T} & 0 & 0 \\
0 & 0 & \mathbf{1}_{16}
\end{array}\right)
$$

where $W$ is an integer matrix with determinant \pm 1 . In the case without Wilson lines, these transformations are modular whenever $W$ satisfies

$$
W Q=Q^{*} W,
$$

and they generate a subgroup of $\mathcal{G}_{\mathcal{O}}$. The action induced on the backgrounds $(\operatorname{via}(2.13))$ is

$$
\begin{gathered}
g \longrightarrow W \frac{1}{g+b} g \frac{1}{g-b} W^{T}, \\
b \longrightarrow-W \frac{1}{g+b} b \frac{1}{g-b} W^{T} .
\end{gathered}
$$

In the case when there is a non-vanishing Wilson line this transformation is not modular, and no similar general expression for a modular duality-type symmetry is known.

There is also a number of so called axionic shift symmetries, generated by

$$
\Omega_{T}:=\left(\begin{array}{ccc}
\mathbf{1}_{d} & 0 & 0 \\
\epsilon & \mathbf{1}_{d} & 0 \\
0 & 0 & \mathbf{1}_{16}
\end{array}\right),
$$

where $\epsilon$ is an antisymmetric integer matrix. The corresponding action on the background is $b \longrightarrow b+\epsilon$. Generically a number of these symmetries remain modular on the orbifold; in fact any axionic modulus may be shifted by an integer.

Moduli spaces of supersymmetric compactifications posess a complex structure, which means in particular that one can define complex coordinates on moduli space in a natural way, and many quantities will take a simple form in these variables. Already in the case of two dimensional orbifolds [19, 20], which posess two real moduli, it is clearly of great advantage to express modular symmetries in terms of a single complex modulus. In models with more parameters this advantage becomes a prerequisite, if one is interested in obtaining any useful formulae at all. 


\section{The duality group for the $Z_{7}$ orbifold}

In this section we discuss in detail the $Z_{7}$ orbifold, which can be defined as a Coxeter twist [12] in the root lattice of $S U(7)$ and deformations thereof. The twist matrix in the lattice basis can be chosen as

$$
Q=\left(\begin{array}{llllll}
0 & 0 & 0 & 0 & 0 & -1 \\
1 & 0 & 0 & 0 & 0 & -1 \\
0 & 1 & 0 & 0 & 0 & -1 \\
0 & 0 & 1 & 0 & 0 & -1 \\
0 & 0 & 0 & 1 & 0 & -1 \\
0 & 0 & 0 & 0 & 1 & -1
\end{array}\right)
$$

and $\mathcal{R}$ is given by (3.5), (3.6) with $\delta=0$ and $a=0$. The solutions to (3.9)-(3.10) read

$$
g=\left(\begin{array}{rrrrrr}
2 g_{0} & g_{1} & g_{2} & g_{3} & g_{3} & g_{2} \\
g_{1} & 2 g_{0} & g_{1} & g_{2} & g_{3} & g_{3} \\
g_{2} & g_{1} & 2 g_{0} & g_{1} & g_{2} & g_{3} \\
g_{3} & g_{2} & g_{1} & 2 g_{0} & g_{1} & g_{2} \\
g_{3} & g_{3} & g_{2} & g_{1} & 2 g_{0} & g_{1} \\
g_{2} & g_{3} & g_{3} & g_{2} & g_{1} & 2 g_{0}
\end{array}\right)
$$

with the constraint $\sum_{i=0}^{3} g_{i}=0$, and

$$
b=\left(\begin{array}{rrrrrr}
0 & b_{1} & b_{2} & b_{3} & -b_{3} & -b_{2} \\
-b_{1} & 0 & b_{1} & b_{2} & b_{3} & -b_{3} \\
-b_{2} & -b_{1} & 0 & b_{1} & b_{2} & b_{3} \\
-b_{3} & -b_{2} & -b_{1} & 0 & b_{1} & b_{2} \\
b_{3} & -b_{3} & -b_{2} & -b_{1} & 0 & b_{1} \\
b_{2} & b_{3} & -b_{3} & -b_{2} & -b_{1} & 0
\end{array}\right) .
$$

Here any three of the $g_{i}$ can be taken as the (real) metric moduli, and the $b_{i}$ are the (real) axionic moduli.

In the complex spacetime basis, where the complex moduli will be defined, the twist is simply given by

$$
\theta=\operatorname{diag}\left(\alpha, \alpha^{-1}, \alpha^{2}, \alpha^{-2}, \alpha^{3}, \alpha^{-3}\right),
$$

where $\alpha \equiv \exp (2 \pi i / 7)$. The transformation matrix $e$, relating the two bases via

$$
\theta e=e Q
$$


is given by

$$
e=\left(\begin{array}{llllll}
\alpha^{2} & \alpha^{3} & \alpha^{4} & \alpha^{5} & \alpha^{6} & 1 \\
\alpha^{5} & \alpha^{4} & \alpha^{3} & \alpha^{2} & \alpha & 1 \\
\alpha^{4} & \alpha^{6} & \alpha & \alpha^{3} & \alpha^{5} & 1 \\
\alpha^{3} & \alpha & \alpha^{6} & \alpha^{4} & \alpha^{2} & 1 \\
\alpha^{6} & \alpha^{2} & \alpha^{5} & \alpha & \alpha^{4} & 1 \\
\alpha & \alpha^{5} & \alpha^{2} & \alpha^{6} & \alpha^{3} & 1
\end{array}\right)
$$

The background fields in the complex spacetime basis are denoted by $G$ and $B$. They are connected with their lattice basis counterparts by the equations

$$
G \pm B=e^{*}(g \pm b) e^{-1} .
$$

From this one can read off the complex moduli. The result is

$$
\begin{array}{ll}
t_{1}=-i \tan \left(\frac{\pi}{7}\right) & {\left[g_{1}+\frac{s_{1}^{2}}{s_{3}^{2}} g_{2}+\frac{s_{2}^{2}}{s_{3}^{2}} g_{3}\right]+b_{1}+\frac{s_{2}}{s_{1}} b_{2}+\frac{s_{3}}{s_{1}} b_{3},} \\
t_{2}=-i \tan \left(\frac{2 \pi}{7}\right) & {\left[g_{1}+\frac{s_{2}^{2}}{s_{1}^{2}} g_{2}+\frac{s_{3}^{2}}{s_{1}^{2}} g_{3}\right]+b_{1}-\frac{s_{3}}{s_{2}} b_{2}-\frac{s_{1}}{s_{2}} b_{3},} \\
t_{3}=-i \tan \left(\frac{3 \pi}{7}\right) & {\left[g_{1}+\frac{s_{3}^{2}}{s_{2}^{2}} g_{2}+\frac{s_{1}^{2}}{s_{2}^{2}} g_{3}\right]+b_{1}-\frac{s_{1}}{s_{3}} b_{2}+\frac{s_{2}}{s_{3}} b_{3},}
\end{array}
$$

where

$$
\begin{aligned}
& s_{k} \equiv \sin (2 \pi k / 7), \\
& c_{k} \equiv \cos (2 \pi k / 7) .
\end{aligned}
$$

The formulae (4.8) involve a choice of normalization, which will be explained below. The requirement that the metric be positive definite here translates into the statement that each complex modulus takes values in the complex upper half-plane.

The generators of the axionic shifts $T_{k}$ are defined as shifts of the real moduli $b_{i}$ leading trivially to modular symmetries. Thus one has

$$
T_{k}\left(t_{m}\right)=t_{m}+a_{k m}
$$

with

$$
a_{k m}=\left(\begin{array}{ccc}
1 & 1 & 1 \\
s_{2} / s_{1} & -s_{3} / s_{2} & -s_{1} / s_{3} \\
s_{3} / s_{1} & -s_{1} / s_{2} & s_{2} / s_{3}
\end{array}\right) .
$$

The first row in $a_{k m}$ shows that one of the axionic shifts acts on the complex moduli in the usual way. This in fact motivated the normalization chosen in (4.8).

At this point is is interesting to check whether one can find combinations of the axionic shifts which would be decoupled on the $t_{k}$, as would be necessary if $S L(2 ; \mathbf{Z})^{3}$ were to be realized here. Consider shifting the $b_{i}$ by $k_{i}$,

\footnotetext{
${ }^{3}$ When working with a complex basis one has to use the hermitian conjugate instead of transposition. Thus the star now denotes the inverse of the hermitian conjugate.
} 
where $k_{i}$ are integers. One would like to be able to find a choice of these integers such that under the above transformation $t_{1}$ would undergo a unit shift, but the remaining moduli would be invariant (and similarly for $t_{2}$ and $t_{3}$ ). For this to happen one has to solve a system of three linear equations which follow from (4.8) and the above requirements. This system of equations has no integer solutions. This shows, that on the complex moduli associated with the complex planes it is not possible to define an $S L(2 ; \mathbf{Z})^{3}$.

Having found the overall shift symmetry $T_{1}$ we have to answer the question, whether there is a related duality transformation $S_{1}$ transforming all $t_{i}$ into their negative inverses and so completing an overall $S L(2, \mathbf{Z})$. This is indeed the case and the corresponding $W$ matrix satisfying condition (3.15) is

$$
W_{1}=\left(\begin{array}{rrrrrr}
0 & -1 & 0 & 0 & 0 & 0 \\
1 & 0 & -1 & 0 & 0 & 0 \\
0 & 1 & 0 & -1 & 0 & 0 \\
0 & 0 & 1 & 0 & -1 & 0 \\
0 & 0 & 0 & 1 & 0 & -1 \\
0 & 0 & 0 & 0 & 1 & 0
\end{array}\right)
$$

It will be shown below that it is also possible to find symmetry transformations $S_{2}$ and $S_{3}$ serving as the $S L(2, \mathbf{Z})$ "partners" of $T_{2}$ and $T_{3}$, i.e.

$$
S_{k}^{2}=1, \quad\left(S_{k} T_{k}\right)^{3}=1 .
$$

It is important to emphasize, however, that the transformations $S_{k}$ do not mutually commute. This can be checked by studying the explicit transformation rules on the complex moduli as given later on.

The condition (3.11) for group elements $\Omega \in \mathcal{G}_{\mathcal{O}}$ involves the discrete parameter $p$. The transformations described above were all solutions with $p=1$ and they were all realized holomorphically on the moduli. The solutions with $p \neq 1$ lead to transformations which are non-holomorphic [25]. In these cases the matrices $\Omega$, although commuting with the projector onto twist invariant states, do not commute with the defining matrix $\mathcal{R}$. For our $Z_{7}$ case we could have chosen equivalently any power $Q^{n}$ with $n \neq 0 \bmod 7$ to be the defining twist and solutions with non-trivial $p$ just correspond to exchanging different twist powers. As for the moduli, they are in general permuted and complex conjugated. If one chooses such a transformation $R$ for $p=3$ or $p=5$, then one can generate all the other ones with non-trivial $p$ by combining $R$ with the transformations of the holomorphic subgroup.

In the present case we find $R$ in the form

$$
\Omega_{R}:=\left(\begin{array}{ccc}
V & 0 & 0 \\
0 & V^{*} & 0 \\
0 & 0 & \mathbf{1}_{16}
\end{array}\right)
$$


with

$$
V=\left(\begin{array}{llllll}
0 & 0 & 0 & 1 & 0 & -1 \\
0 & 1 & 0 & 0 & 0 & -1 \\
0 & 0 & 0 & 0 & 0 & -1 \\
0 & 0 & 0 & 0 & 1 & -1 \\
0 & 0 & 1 & 0 & 0 & -1 \\
1 & 0 & 0 & 0 & 0 & -1
\end{array}\right)
$$

satisfying

$$
V Q=Q^{3} V
$$

The resulting transformation on the moduli reads

$$
R:\left(t_{1}, t_{2}, t_{3}\right) \rightarrow\left(-2 c_{1} \bar{t}_{2},-2 c_{2} t_{3},-2 c_{3} t_{1}\right) .
$$

The essential property of $R$ is that

$$
\begin{aligned}
& R T_{1} R^{-1}=T_{3}, \\
& R T_{3} R^{-1}=T_{2},
\end{aligned}
$$

i.e., by conjugating $T_{1}$ with $R$ one obtains the other axionic shift symmetries. Observe also that

$$
R^{3}:\left(t_{1}, t_{2}, t_{3}\right) \rightarrow\left(-\overline{t_{1}},-\overline{t_{2}},-\overline{t_{3}}\right) .
$$

Thus $R^{3}$ gives rise to a charge conjugation symmetry.

It is clear that the transformations

$$
\begin{aligned}
S_{2} & :=R^{2} S_{1} R^{-2}, \\
S_{3} & :=R S_{1} R^{-1},
\end{aligned}
$$

satisfy the relations (4.13). The transformations $S_{k}$ act on the moduli according to

$$
S_{k}\left(t_{m}\right)=p_{k m} / t_{m}
$$

where the numbers $p_{k m}$ are given by

$$
\left(p_{k m}\right)=\left(\begin{array}{ccc}
-1 & -1 & -1 \\
-4 c_{1}^{2} & -4 c_{2}^{2} & -4 c_{3}^{2} \\
\frac{-1}{4 c_{3}^{2}} & \frac{-1}{4 c_{1}^{2}} & \frac{-1}{4 c_{2}^{2}}
\end{array}\right) .
$$

From this it is easy to see that different $S$ transformations and thus the corresponding $S L(2 ; \mathbf{Z})$ groups do not commute. Note, that the composition of two different duality transformations yields a simultaneous rescaling of all moduli, e.g.

$$
S_{1} S_{2}:\left(t_{1}, t_{2}, t_{3}\right) \rightarrow\left(\frac{t_{1}}{4 c_{1}^{2}}, \frac{t_{2}}{4 c_{2}^{2}}, \frac{t_{3}}{4 c_{3}^{2}}\right),
$$


and that this is an infinite order symmetry. By composing such elements with $S_{1}, T_{1}$ one can construct an infinite number of non-commuting $S L(2 ; \mathbf{Z})$ groups.

In summary, we found three generators $S:=S_{1}, T:=T_{1}$ and $R$ satisfying a number of relations. The basic ones are given by

$$
\begin{gathered}
S^{2}=(S T)^{3}=R^{6}=1, \\
(T R)^{6}=\left(T R^{3}\right)^{2}=\left(S R^{3}\right)^{2}=1, \\
\left(S R T R^{-1}\right)^{7}=1 .
\end{gathered}
$$

These relations define a group which does not contain $S L(2, \mathbf{Z})^{3}$.

Given an arbitrary string of transformations generated by $S, T$ and $R$ one can always permute all factors of $R$ to one side obtaining a product of a power of $R$ with a purely holomorphic transformation. For a classification we can thus concentrate on the holomorphic $(p=1)$ symmetry transformations. The price to be paid is, however, that the number of holomorphic generators is larger: one has to take all six $S_{i}, T_{i}$.

We now proceed to characterize matrices $\Omega$ which belong to $\tilde{\mathcal{G}}_{\mathcal{O}}$. Recall that such matrices must satisfy two conditions: the invariance condition (2.12), and the compatibility relation (3.11), which we consider for $p=1$. The latter is a linear equation for $\Omega$ and can readily be solved to give a form of $\Omega$ in terms of a number of integer parameters. This can then be substituted into (2.12), which gives a set of quadratic equations for the integer parameters. Some of these equations can be solved, while further parameters may be eliminated by observing that certain $\Omega$ 's act trivially on the moduli. One is then left with a number of quadratic relations between the remaining integers.

To see the mechanics of this procedure let us first apply it to the example of the $Z_{3}$ orbifold in two compact dimensions (without Wilson lines), where the group $\tilde{\mathcal{G}}_{\mathcal{O}}$ is known to be $S L(2 ; \mathbf{Z})$ [19]. The twist in the lattice basis can be taken in the form

$$
Q=\left(\begin{array}{ll}
0 & -1 \\
1 & -1
\end{array}\right),
$$

and $\mathcal{R}$ is given by (3.5), (3.6) with $\delta=0$ and $a=0$. The twist matrix in the complex basis reads

$$
\theta=\operatorname{diag}\left(\alpha, \alpha^{-1}\right)
$$

where now $\alpha=\exp (2 \pi i / 3)$. Since there is no Wilson line in this example, the gauge lattice will be dropped in the formulae given below. 
The solution to (3.11) can be written in the form

$$
\Omega=\left(\begin{array}{rrrr}
u_{3}-u_{5} & -u_{5} & u_{6} & -u_{1}-u_{6} \\
u_{5} & u_{3} & u_{1} & u_{6} \\
u_{7} & u_{4}+u_{7} & u_{2}+u_{8} & -u_{8} \\
-u_{4} & u_{7} & u_{8} & u_{2}
\end{array}\right)
$$

depending on eight integers $u_{i}$. To reduce this further it is convenient to pass to the complex basis. As before, the basis transformation matrix $e$ is defined by (4.5). In this case it reads

$$
e=\left(\begin{array}{ll}
\alpha^{2} & 1 \\
\alpha & 1
\end{array}\right)
$$

It is easy to see that $\Omega$ must be transformed according to

$$
\tilde{\Omega}=E^{*} \Omega E^{T},
$$

where $E$ is the block diagonal matrix

$$
E=\left(\begin{array}{cc}
e & 0 \\
0 & e^{*}
\end{array}\right)
$$

One finds

$$
\tilde{\Omega}=\left(\begin{array}{llll}
z_{1} & 0 & z_{2} & 0 \\
0 & \bar{z}_{1} & 0 & \bar{z}_{2} \\
z_{3} & 0 & z_{4} & 0 \\
0 & \bar{z}_{3} & 0 & \bar{z}_{4}
\end{array}\right)
$$

where

$$
\begin{aligned}
& z_{1}=u_{3}+\alpha u_{5} \\
& z_{2}=\frac{1}{3}\left((1+2 \alpha) u_{1}+(2+\alpha) u_{6}\right) \\
& z_{3}=-(1+2 \alpha) u_{4}+(1-\alpha) u_{7} \\
& z_{4}=u_{2}-\alpha^{2} u_{8}
\end{aligned}
$$

It is useful to look at this from the following point of view: write

$$
\tilde{\Omega}=\left(\begin{array}{ll}
a & b \\
c & d
\end{array}\right),
$$

where $a, b, c, d$ are $2 \times 2$ blocks of the form

$$
x=r_{x}\left(\begin{array}{cc}
\exp i \phi_{x} & 0 \\
0 & \exp -i \phi_{x}
\end{array}\right) .
$$

The quantities $r_{x}$ and $\phi_{x}$ will be referred to as the radius and the phase of a block. It turns out that the phases can be determined, as will now be shown. 
The condition (2.12) in the complex basis implies

$$
\begin{aligned}
& a^{\dagger} c+c^{\dagger} a=0, \\
& b^{\dagger} d+d^{\dagger} b=0, \\
& a^{\dagger} d+c^{\dagger} b=1 .
\end{aligned}
$$

From $(4.36)-4.37)$ it follows that

$$
\begin{aligned}
& \phi_{c}=\phi_{b}, \\
& \phi_{a}=\phi_{d},
\end{aligned}
$$

and

$$
\phi_{a}-\phi_{c}= \pm \frac{\pi}{2}
$$

This way three of the four phases are fixed. The remaining one is fixed by observing that multiplying $\tilde{\Omega}$ by a matrix of the form

$$
\Lambda=\operatorname{diag}(\exp i \psi, \exp -i \psi, \exp i \psi, \exp -i \psi)
$$

for any $\psi$ does not change the transformation induced by $\tilde{\Omega}$ on the moduli. This allows us to set one of the phases (say $\phi_{a}$ ) equal to zero. Thus all phases have been found by using the equation (2.12) and eliminating the redundancy in $\Omega$. Only the four radii remain.

The conditions on the blocks in (4.33) can be stated in the form

$$
\begin{gathered}
\operatorname{Re}(b)=\operatorname{Re}(c)=0, \\
\operatorname{Im}(a)=\operatorname{Im}(d)=0 .
\end{gathered}
$$

The arguments given above can be used to reduce the number of integer parameters in (4.27). One simply enforces the equations (4.42) on $\tilde{\Omega}$. Looking at (4.31) - (4.32) one sees that $u_{i}(i=5, \ldots, 8)$ must be set to zero, so only four integer parameters remain. Back in the lattice basis $\Omega$ reads

$$
\Omega=\left(\begin{array}{rrrr}
u_{3} & 0 & 0 & -u_{1} \\
0 & u_{3} & u_{1} & 0 \\
0 & u_{4} & u_{2} & 0 \\
-u_{4} & 0 & 0 & u_{2}
\end{array}\right)
$$

and the constraint (2.12) gives

$$
u_{1} u_{4}-u_{2} u_{3}=1
$$

which is the standard determinant condition of $S L(2 ; \mathbf{Z})$.

Using (4.25) in (3.9)-(3.10) gives the backgrounds

$$
g=R^{2}\left(\begin{array}{rr}
2 & -1 \\
-1 & 2
\end{array}\right)
$$




$$
b=\left(\begin{array}{rr}
0 & x \\
-x & 0
\end{array}\right),
$$

in terms of real moduli $R^{2}$ and $x$. Passing to the complex basis one finds the complex modulus

$$
\lambda=-i \sqrt{3} R^{2}+x
$$

One may now check, that the $\Omega$ given in (4.43) induces the standard action of $S L(2 ; \mathbf{Z})$ on the complex modulus $\lambda$ :

$$
\lambda \longrightarrow \lambda^{\prime}=\frac{u_{1}+\lambda u_{2}}{u_{3}+\lambda u_{4}}
$$

The arguments given above are easily generalized to the $Z_{7}$ orbifold. Solutions to (3.11) depend in this case on 24 integers. The matrix $\tilde{\Omega}$ now has the form

$$
\tilde{\Omega}=\left(\begin{array}{cccccc}
a_{1} & 0 & 0 & b_{1} & 0 & 0 \\
0 & a_{2} & 0 & 0 & b_{2} & 0 \\
0 & 0 & a_{3} & 0 & 0 & b_{3} \\
c_{1} & 0 & 0 & d_{1} & 0 & 0 \\
0 & c_{2} & 0 & 0 & d_{2} & 0 \\
0 & 0 & c_{3} & 0 & 0 & d_{3}
\end{array}\right)
$$

where $a_{i}, b_{i}, c_{i}, d_{i}$ are $2 \times 2$ blocks of the form (4.34). We thus have 12 radii and 12 phases.

The condition (2.12) for $\tilde{\Omega}$ implies

$$
\begin{gathered}
a_{k}^{\dagger} c_{k}+c_{k}^{\dagger} a_{k}=0 \\
b_{k}^{\dagger} d_{k}+d_{k}^{\dagger} b_{k}=0 \\
a_{k}^{\dagger} d_{k}+c_{k}^{\dagger} b_{k}=1
\end{gathered}
$$

It follows from this, that

$$
\begin{aligned}
& \operatorname{Arg}\left(a_{k}\right)=\operatorname{Arg}\left(d_{k}\right), \\
& \operatorname{Arg}\left(b_{k}\right)=\operatorname{Arg}\left(c_{k}\right),
\end{aligned}
$$

and

$$
\operatorname{Arg}\left(a_{k}\right)=\operatorname{Arg}\left(c_{k}\right) \pm \pi / 2,
$$

where $\operatorname{Arg}(x)$ denotes the phase of the block $x$. The above equations determine 9 of the 12 phases in terms of 3, which can however be chosen arbitrarily, using the freedom to multiply $\tilde{\Omega}$ by the following matrix, which induces a trivial transformation on the moduli:

$$
\Lambda=\operatorname{diag}\left(\lambda_{1}, \lambda_{2}, \lambda_{3}, \lambda_{1}, \lambda_{2}, \lambda_{3}\right)
$$


Here the $\lambda_{i}$ are diagonal $2 \times 2$ blocks

$$
\lambda_{k}=\operatorname{diag}\left(\exp i \psi_{k}, \exp -i \psi_{k}\right)
$$

and the $\psi_{k}$ are arbitrary.

Thus one can choose $a_{k}, d_{k}$ to be real, and $b_{k}, c_{k}$ to be purely imaginary. Imposing this in $\tilde{\Omega}$ leaves 12 integers, which must satisfy quadratic constraints following From (2.12), in analogy with (4.44).

The result of the calculation described above is as follows. The matrix $\Omega$ depends on 12 integers denoted by $u_{i}$, and is given in the appendix. The quadratic constraints read

$$
\begin{array}{r}
u_{5} u_{7}+u_{4} u_{8}+u_{6} u_{8}-u_{4} u_{9}-u_{1} u_{10}-u_{1} u_{11}+u_{3} u_{11}-u_{2} u_{12}=(4.57) \\
u_{6} u_{7}+u_{5} u_{8}+u_{4} u_{9}-u_{6} u_{9}-u_{2} u_{10}+u_{3} u_{10}-u_{1} u_{11}-u_{1} u_{12}+u_{2} u_{12}=(4.58) \\
u_{4} u_{7}-u_{4} u_{8}+u_{5} u_{8}-u_{5} u_{9}+u_{6} u_{9}-u_{1} u_{10}+u_{2} u_{10}-u_{2} u_{11}+u_{3} u_{12}=(14.59)
\end{array}
$$

It is possible to show, that the transformations induced by such matrices transform the complex moduli according to

$$
t_{k} \longrightarrow t_{k}{ }^{\prime}=\frac{a_{k}+b_{k} t_{k}}{c_{k}+d_{k} t_{k}}
$$

where the parameters $a_{k}, b_{k}, c_{k}, d_{k}$ can be expressed in terms of the integers $u_{i}$.

\section{Quantized Wilson lines and duality}

In this section we will come to the question, whether non-trivial (quantized) Wilson lines can change the results obtained so far. At first sight it might appear that these Wilson lines essentially affect only the gauge lattice, since on one hand they break the gauge group while on the other hand they do not seem to change the twist in the space part which stays in its symmetric form. However, as explained in section 2, this is not quite the case, since quantized Wilson lines are not just usual background parameters like the moduli. Recall, that the corresponding defining matrix mixes up all types of quantum numbers. Indeed, in [17] it was shown, that naive generalizations of duality lead to the class of asymmetric orbifolds, thus not representing modular symmetries. Consequently, it is interesting to ask whether one can define some kind of duality and, motivated from the results of the preceding section, what role is played by the group $S L(2, \mathbf{Z})$ in these cases.

In order to illustrate what happens, we discuss two simple examples [25]. Thereby we will for simplicity assume that the shift vector (gauge embedding of the point group) acts in one $E_{8}$ and the Wilson line (gauge embedding of 
the space group) is connected to the other one. Then we can disregard the former one as well as the world sheet superfermions which also play a passive role for our discussion.

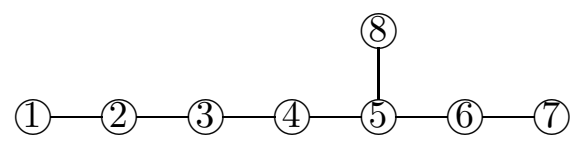

Figure 1: Dynkin diagram of $E_{8}$

We start with the one-dimensional case often referred to as the orbicircle, where the twist action is given by negation of winding and momentum. If we assign the gauge quantum numbers according to figure 1 and use the Wilson linet

$$
a=\frac{1}{2}(0,0,0,0,0,0,1,1)
$$

then we find the right moving Narain momentum

$$
P_{R}=\frac{1}{2} m-\frac{1}{2 \alpha^{\prime}} r^{2} n+\frac{1}{4}\left(l_{5}+l_{6}-n\right)-\frac{1}{2}\left(l_{7}+l_{8}\right),
$$

where we reinserted the dimensionful parameter $\alpha^{\prime}$. Notice the factor of $1 / 4$ in this expression, which has no counterpart in the case of vanishing Wilson line. Motivated by this we rewrite (5.62) as

$$
P_{R}=-\frac{2}{\alpha^{\prime}} r^{2}\left[\frac{1}{4} n-\frac{\alpha^{\prime}}{8 r^{2}}\left(2 m-n+l_{5}+l_{6}-2 l_{7}-2 l_{8}\right)\right] .
$$

From dimensional considerations we conclude that the expression in parenthesis gives the new winding. Recalling that scalar products are built with the inverse metric we find invariance of $P_{R}^{2}$ when transforming

$$
\begin{aligned}
r & \rightarrow \frac{\alpha^{\prime}}{2 r}, \\
n & \leftrightarrow 2 m-n+l_{5}+l_{6}-2 l_{7}-2 l_{8}=2 m-n-2 a^{T} C l .
\end{aligned}
$$

The remaining task is to extend this to a complete $O(9,1 ; \mathbf{Z})$ transformation, which can be done with

$$
\begin{aligned}
m & \rightarrow-m, \\
l & \rightarrow l-2 a l .
\end{aligned}
$$

This shows that there is indeed a $Z_{2}$ symmetry in moduli space, which can be interpreted as a generalized duality transformation. Note, however, that

\footnotetext{
${ }^{4} \mathrm{~A}$ non-trivial Wilson line with only one entry would not satisfy $a^{2}=a^{T} C a \in \mathbf{Z}$ necessary for level matching.
} 
the radius is no longer transformed like in the case with vanishing $a$, where $r \rightarrow \frac{\alpha^{\prime}}{r}$. We see that the self-dual radius has changed. This is in perfect agreement with the results in [17], where a simple model with a discrete antisymmetric background was presented and anticipated that these kinds of quantized background fields in fact stand on the same footing.

We will now discuss the example of the $Z_{3}$-orbifold, where in case of vanishing Wilson lines the modular group is known [24 to be $S U(3,3 ; \mathbf{Z})$. This model admits up to three independent Wilson lines 22 and in using two ones one can construct models with three generations of $S U(3) \times S U(2) \times$ $U(1)^{n}$ [26]. Here, again for simplicity, we will restrict ourselves to one independent Wilson line,

$$
a_{1}=a_{2}=\frac{1}{3}(1,0,0,0,0,0,1,1)
$$

where already the important new features occur. This means that we deal effectively with a two-dimensional $Z_{3}$-orbifold, where the modular group for $a=0$ is $P S L(2, \mathbf{Z})$ [20].

The twist matrix in this case is given by (4.25) and we obtain the right moving Narain momentum

$$
\begin{aligned}
P_{R i} & =\frac{1}{2} m_{i}-\frac{1}{2 \alpha^{\prime}}(g+b)_{i j} n^{j}+\frac{1}{6}\left(l_{2}+l_{5}+l_{6}-n^{1}-n^{2}\right)-\frac{1}{3}\left(l_{1}+l_{7}+l_{8}\right) \\
& =\frac{1}{2} m_{i}-\frac{1}{2 \alpha^{\prime}}(g+b)_{i j} n^{j}+\frac{1}{6} t_{i}
\end{aligned}
$$

where we introduced the vector $t$ with $\left(a_{1}=a_{2}\right)$ equal components $t_{1}=t_{2} \in$ Z. In analogy to the previous example we rewrite this to

$$
P_{R}=-\frac{3}{\alpha^{\prime}}(g+b) W^{T}\left[\frac{1}{6} W^{*} n-W^{*} \frac{\alpha^{\prime}}{18(g+b)} W^{-1}(3 W m+W t)\right],
$$

where as in section 2 we introduced a matrix $W$. Consequently we try the following ansatz for duality:

$$
\begin{aligned}
\hat{g}+\hat{b} & =W^{*} \frac{\alpha^{\prime 2}}{9(g+b)} W^{-1}, \\
\hat{n} & =W(3 m+t), \\
3 \hat{m}+\hat{t} & =W^{*} n, \\
\hat{P}_{R} & =-W^{*} \frac{\alpha^{\prime}}{3(g+b)},
\end{aligned}
$$

where we chose to indicate the transformed quantities by hats. Notice the factor of 3 multiplying the momenta on the right hand side of (5.70). Thus to make sure that in the transformed theory the quantum number $\hat{n}^{1}+\hat{n}^{2}$ 
runs over all integers we have to require that the sum of matrix elements in $W$ does not vanish mod 3. The solutions to equation (3.15) are given by

$$
\begin{aligned}
& W_{1}=\left(\begin{array}{rr}
0 & 1 \\
-1 & 0
\end{array}\right), \quad W_{2}=\left(\begin{array}{cc}
1 & 0 \\
1 & 1
\end{array}\right), \quad W_{3}=\left(\begin{array}{ll}
1 & 1 \\
0 & 1
\end{array}\right), \\
& W_{4}=\left(\begin{array}{rr}
1 & 0 \\
0 & -1
\end{array}\right), \quad W_{5}=\left(\begin{array}{ll}
0 & 1 \\
1 & 1
\end{array}\right), \quad W_{6}=\left(\begin{array}{ll}
1 & 1 \\
1 & 0
\end{array}\right) \text {, }
\end{aligned}
$$

as well as there negatives and in fact none of them meets our requirement. This shows that discrete Wilson lines can break duality symmetry 25]. An immediate conclusion is that the group $\operatorname{PSL}(2, \mathbf{Z})$ is broken as well, a fact with far reaching consequences. This group is well known in the mathematical literature since a long time [27 and this knowledge has been used intensively for the construction of four dimensional low energy field theories [1]. For cases with quantized Wilson lines one obviously has to consider different groups. So let us look for the unbroken symmetry group.

We will try to modify the ansatz (5.70) and in order to circumvent the above problem we add a partial axionic shift, i.e. we shift the momentum quantum number by a non-integer amount, which by itself is not a symmetry:

$$
\hat{n}=W\left(3 m+t+\epsilon_{k / 3} n\right),
$$

with

$$
\epsilon_{k / 3}=\left(\begin{array}{cc}
0 & k \\
-k & 0
\end{array}\right), \quad k \in \mathbf{Z} \notin 3 \mathbf{Z} .
$$

But (5.71) shows that the $m$ transformation is problematic as well, since it follows

$$
3\left(\hat{m}_{1}-\hat{m}_{2}\right)=\left(W_{11}^{*}-W_{21}^{*}\right) n^{1}+\left(W_{12}^{*}-W_{22}^{*}\right) n^{2},
$$

so that the right hand side must be a multiple of three for the ansatz to work. Again this cannot be achieved for any $W$ and we modify the ansatz further to

$$
3 \hat{m}+\hat{t}+\hat{\epsilon}_{\hat{k} / 3} \hat{n}=W^{*} n,
$$

where as in (5.75) we defined $\hat{\epsilon}_{\hat{k} / 3}$ with elements $\hat{k}$. Then (5.76) takes on the form

$$
3\left(\hat{m}_{1}-\hat{m}_{2}\right)=\left(W_{11}^{*}-W_{21}^{*}-\hat{k}\right) n^{1}+\left(W_{12}^{*}-W_{22}^{*}-\hat{k}\right) n^{2} .
$$

Now one can indeed achieve that the r.h.s. is a multiple of 3 and the simplest solution arises for $W_{1}$,

$$
\begin{aligned}
n & \rightarrow U n+3 W_{1} m-3 \sigma_{3} a^{T} C l, \\
m & \rightarrow U^{*} m, \\
l & \rightarrow l+3 a \sigma_{3} m,
\end{aligned}
$$

\footnotetext{
${ }^{5}$ See reference [25] for more details.
} 
with $\sigma_{3}$ the Pauli matrix and

$$
U=\left(\begin{array}{rr}
0 & -1 \\
1 & 2
\end{array}\right)
$$

The above construction involves an inversion $\sigma$ and partial shifts $\tau$. We normalize the complex modulus

$$
\lambda:=\frac{3}{\alpha^{\prime}}(x+i \sqrt{\operatorname{det} g})
$$

in such a way that we have

$$
\begin{aligned}
\sigma: & & \lambda \rightarrow-\frac{1}{\lambda}, \\
\tau: & & \lambda \rightarrow \lambda+1 .
\end{aligned}
$$

This notation shows that the modular group at hand is the subgroup of $P S L(2, \mathbf{Z})$ generated by

$$
\begin{aligned}
& A:=\tau \sigma \tau, \\
& B:=\tau^{3},
\end{aligned}
$$

where $B$ refers to the usual axionic shift symmetry which is clearly also present. In matrix notation wie can represent the action of $A$ as

$$
\hat{g}+\hat{b}-\frac{\alpha^{\prime}}{3} \epsilon_{1 / 3}=W^{*} \frac{\alpha^{\prime 2}}{9\left(g+b+\frac{\alpha^{\prime}}{3} \epsilon_{1 / 3}\right)} W^{-1},
$$

and the complex modulus transforms as

$$
\lambda \rightarrow \frac{\lambda}{\lambda+1}
$$

With the help of (5.83) one easily finds the relation

$$
(A B)^{3}=1
$$

whereas $A$ no longer squares to one as would be necessary for $\operatorname{PSL}(2, \mathbf{Z})$. It can be shown 25] that for the ordinary $Z_{3}$ orbifold there exists another symmetry generator $C$ with a non-holomorphic action,

$$
C: \quad \lambda \rightarrow-\bar{\lambda},
$$

giving rise to an exchange of particles with antiparticles in the twisted sector. It also survives in the Wilson line case and with the help of

$$
\tau C \tau=C
$$

\footnotetext{
${ }^{6}$ The quantities $g$ and $x$ are defined in 4.45$)-(4.46$ ).
} 
one readily proves the relations [25]

$$
C^{2}=(A C)^{2}=(B C)^{2}=1 .
$$

Note, that none of the order two operations above can be utilized to define a new kind of duality transformation, since all of them posess fixed curves instead of fixed points as is the case for duality. Similarly, no $\operatorname{PSL}(2, \mathbf{Z})$ subgroup can reappear. Notice also, that the relations (5.89) can be used to commute all generators $C$ to one side of a given string of operations showing that $C$ corresponds to a $Z_{2}$ factor commuting with the holomorphic part of the modular group.

Having established the breakdown of duality in this example, we are confronted with the question whether there is still an operation (1) possessing fixed points and (2) mapping small radii to large ones. Whereas $A$ leaves fixed only the origin, which does not belong to the upper half-plane, the combination $A B$ does in fact the job. It has a fixed point at $\sqrt{3} e^{i \pi / 6}$ and property (2) is obvious from the construction.

\section{Acknowledgements}

We would like to thank Hans-Peter Nilles for many enlightening discussions on this and related topics. We also thank Stefan Theisen for helpful comments. A large part of this work was done while M.S. was in München as a fellow of the Alexander von Humboldt-Stiftung.

\section{A Appendix}

For typographical reasons it is convenient to present the matrix $\Omega$ discussed in section 4 in block form:

$$
\Omega=\left(\begin{array}{cc}
A & B \\
C & D
\end{array}\right)
$$

where the blocks are given by

$$
A=\left(\begin{array}{rrrrrr}
u_{3} & 0 & u_{2} & u_{1} & u_{1} & u_{2} \\
-u_{2} & -u_{2}+u_{3} & -u_{2} & 0 & u_{1}-u_{2} & u_{1}-u_{2} \\
-u_{1}+u_{2} & -u_{1} & -u_{1}+u_{3} & -u_{1} & -u_{1}+u_{2} & 0 \\
0 & -u_{1}+u_{2} & -u_{1} & -u_{1}+u_{3} & -u_{1} & -u_{1}+u_{2} \\
u_{1}-u_{2} & u_{1}-u_{2} & 0 & -u_{2} & -u_{2}+u_{3} & -u_{2} \\
u_{2} & u_{1} & u_{1} & u_{2} & 0 & u_{3}
\end{array}\right)
$$




$$
\begin{aligned}
& B=\left(\begin{array}{rrrrrr}
0 & -u_{6} & -u_{5} & -u_{4} & u_{4} & u_{5} \\
u_{6} & 0 & -u_{6} & -u_{5} & -u_{4} & u_{4} \\
u_{5} & u_{6} & 0 & -u_{6} & -u_{5} & -u_{4} \\
u_{4} & u_{5} & u_{6} & 0 & -u_{6} & -u_{5} \\
-u_{4} & u_{4} & u_{5} & u_{6} & 0 & -u_{6} \\
-u_{5} & -u_{4} & u_{4} & u_{5} & u_{6} & 0
\end{array}\right) \\
& C=\left(\begin{array}{rrrrrr}
0 & -u_{9} & -u_{8} & -u_{7} & -u_{8} & -u_{9} \\
u_{9} & 0 & -u_{8} & -u_{7}-u_{8}+u_{9} & -u_{7}-u_{8}+u_{9} & -u_{8} \\
u_{8} & u_{8} & 0 & -u_{7} & -u_{7}-u_{8}+u_{9} & -u_{7} \\
u_{7} & u_{7}+u_{8}-u_{9} & u_{7} & 0 & -u_{8} & -u_{8} \\
u_{8} & u_{7}+u_{8}-u_{9} & u_{7}+u_{8}-u_{9} & u_{8} & 0 & -u_{9} \\
u_{9} & u_{8} & u_{7} & u_{8} & u_{9} & 0
\end{array}\right) \\
& D=\left(\begin{array}{rrrrrr}
u_{12} & u_{11} & u_{10} & 0 & -u_{10} & -u_{11} \\
0 & u_{11}+u_{12} & u_{10}+u_{11} & u_{10} & -u_{10} & -u_{10}-u_{11} \\
-u_{11} & u_{11} & u_{10}+u_{11}+u_{12} & u_{10}+u_{11} & 0 & -u_{10}-u_{11} \\
-u_{10}-u_{11} & 0 & u_{10}+u_{11} & u_{10}+u_{11}+u_{12} & u_{11} & -u_{11} \\
-u_{10}-u_{11} & -u_{10} & u_{10} & u_{10}+u_{11} & u_{11}+u_{12} & 0 \\
-u_{11} & -u_{10} & 0 & u_{10} & u_{11} & u_{12}
\end{array}\right)
\end{aligned}
$$




\section{References}

[1] R. Dijkgraaf, E. Verlinde and H. Verlinde, Comm. Math. Phys. 115 (1988) 649

[2] M. Dine, N. Seiberg Nucl. Phys. B 301 (1988) 357

[3] N. Seiberg, Nucl. Phys. B 258 (1988) 286

[4] S. Ferrara, D. Lüst, A. Shapere and S. Theisen, Phys. Lett. 225 B (1989) 363

[5] S. Ferrara, D. Lüst and S. Theisen, Phys. Lett. 233 B (1989) 147

[6] S. Ferrara and S. Theisen, Moduli Spaces, Effective Actions and Duality Symmetry in String Compactifications, CERN-TH 5652/90

[7] K. Kikkawa and M. Yamasaki, Phys. Lett. 149B (1984) 357;

N. Sakai and I. Senda, Prog. Theor. Phys. 75 (1984) 692;

[8] A. Shapere and F. Wilczek, Nucl. Phys. B320 (1989) 669

[9] A. Giveon, E. Rabinovici and G. Veneziano, Nucl. Phys. B322 (1989) 167

[10] M. Dine, P. Huet and N. Seiberg, Nucl. Phys. B322 (1989) 301

[11] L. Dixon, J. A. Harvey, C. Vafa and E. Witten, Nucl. Phys. B261 (1985) 678 and Nucl. Phys. B274 (1986) 285

[12] L. E. Ibáñez, J. Mas, H. P. Nilles and F. Quevedo, Nucl. Phys. B301 (1988) 157

[13] K. S. Narain, Phys. Lett. 169 B (1986) 41;

[14] K. S. Narain, M. H. Sarmadi and E. Witten, Nucl. Phys. B279 (1987) 369

[15] M. Spaliński, Nucl. Phys. B B377 (1992) 339

[16] M. Spaliński, Phys. Lett. 275 B (1992) 47

[17] J. Erler, D. Jungnickel and H. P. Nilles, Phys. Lett. 276 B (1992) 303

[18] J. Erler, D. Jungnickel and H. P. Nilles, M. Spaliński, "Duality symmetry and its anomalies", talk at the Int. Workshop on Electroweak Physics Beyond the Standard Model, Valencia, 1991, preprint MPI-PH-91-104. 
[19] W. Lerche, D. Lüst and N. P. Warner, Phys. Lett. 231 B (1989) 417

[20] J. Lauer, J. Mas and H. P. Nilles, Nucl. Phys. B351 (1991) 353

[21] P. Candelas, X. de la Ossa, P. Green and L. Parkes, Nucl. Phys. B359 (1991) 21

[22] L. E. Ibáñez, H. P. Nilles and F. Quevedo, Phys. Lett. 187B (1987) 25

[23] J. Erler and A. Klemm, Comment on the Generation Number in Orbifold Compactifications, preprint TUM-TH-146/92

[24] S. Ferrara, P. Frè and P. Soriani, On the Moduli Space of the $T^{6} / Z_{3}$ Orbifold and its Modular Group, CERN-TH 6364/92 (SISSA 5/92/EP)

[25] J. Erler, "Untersuchung von Moduliraumen in Stringtheorien", Ph. D. thesis at the Technische Universität München; Max-Planck-Institut preprint MPI-Ph/92-21

[26] L. E. Ibáñez, J. E. Kim, H. P. Nilles and F. Quevedo, Phys. Lett. 191B (1987) 282

[27] F. Klein und R. Fricke, Vorlesungen über die Theorie der elliptischen Modulfunktionen, Teubner-Verlag, Leipzig (1890) 